\title{
Towards the Characterization of Max-Resolution Transformations of UCSs by UP-Resilience ${ }^{\star}$
}

\author{
Mohamed Sami Cherif and Djamal Habet \\ Aix Marseille Univ, Université de Toulon, CNRS, LIS, Marseille, France \\ \{mohamed-sami.cherif, djamal.habet\}@univ-amu.fr
}

\begin{abstract}
The Max-SAT problem consists in finding an assignment maximizing the number of satisfied clauses. Complete methods for this problem include Branch and Bound (BnB) algorithms which use maxresolution, the inference rule for Max-SAT, to ensure that every computed Inconsistent Subset (IS) is counted only once in the lower bound estimation. However, learning max-resolution transformations can be detrimental to their performance so they are usually selectively learned if they respect certain patterns. In this paper, we focus on recently introduced patterns called Unit Clause Subsets (UCSs). We characterize the transformations of certain UCS patterns using the UP-resilience property. Finally, we explain how our result can help extend the current patterns.
\end{abstract}

Keywords: Max-resolution · UP-resilience · Unit Clause Subset

\section{Introduction}

Max-SAT is an optimization extension of the satisfiability (SAT) problem. For a given formula in Conjunctive Normal Form (CNF), it consists in finding an assignment of the variables which maximizes the number of satisfied clauses. Complete methods for this problem include SAT based approaches (e.g. MAXHS [7], OPEN-WBO [13], EVA [14], WPM1 [5]) and Branch and Bound (BnB) algorithms (e.g. AHMAXSAT [3], AKMAXSAT [9], MAXSATZ [10,12]) among others. The former which iteratively call SAT solvers are particularly efficient on industrial instances while the latter are competitive on random and crafted instances.

$\mathrm{BnB}$ based approaches construct a search tree and compute, at each node, the Lower Bound (LB) by counting the disjoint Inconsistent Subsets (ISs) of the formula using Simulated Unit Propagation (SUP) [11]. When an IS is found, it is either temporarily deleted or transformed by max-resolution, the inference rule for Max-SAT [6,8], to ensure that it will be counted only once. However, learning max-resolution transformations, i.e., memorizing them in the current subtree (including the current node), may affect negatively the quality of the lower bound estimation $[2,4,12]$. Therefore, state of the art solvers learn transformations selectively mainly in the form of patterns [12].

* This work is funded by the French National Research Agency (ANR), reference ANR-16-C40-0028. 
Recently, new patterns called Unit Clause Subsets (UCSs) were introduced and empirically studied in [2]. The most significant feature of these patterns is producing unit clauses after the transformation by max-resolution. The empirical study of these patterns lead to the first observations on the relation between max-resolution transformations and the efficiency of the SUP mechanism which is indispensable for the lower bound estimation. These observations were formally stated by the introduction of a new property called UP-resilience [4].

In this paper, we conduct a theoretical study of particular UCS patterns and, more specifically, their relation with UP-resilience: we prove that binary UCSs are UP-resilient and we generalize this result on UCSs where only one clause of any size is involved in the conflict. We also explain how our results can help extend the current patterns by showing that the current mechanisms in $\mathrm{BnB}$ solvers can't ensure UP-resilience for these patterns.

This paper is organized as follows. In Section 2, we give basic definitions and notations. In Section 3, we show how the UP-resilience property highlights the impact of max-resolution transformations on the SUP mechanism. We characterize UCS transformations and we show the limit of the current mechanisms in Section 4 and we conclude in Section 5 .

\section{Definitions \& Notations}

Let $X$ be a set of propositional variables. A literal $l$ is a variable $x \in X$ or its negation $\bar{x}$ and a clause is disjunction of literals, represented as a set of literals. A formula in Conjunctive Normal Form (CNF) is a conjunction of clauses and can be represented as a set of clauses. An assignment $I: X \longrightarrow\{$ true, false $\}$ maps each variable to a Boolean value and is represented as a set of literals. For a given literal $l, \operatorname{var}(l)$ denotes the variable appearing in $l$. A clause $c$ is satisfied by an assignment $I$ if at least one of its literals is satisfied, i.e., $\exists l \in c$ such that $l \in I$. The empty clause $\square$ is always falsified. An Inconsistent Subset (IS) of a formula $\Phi$ is an unsatisfiable set of clauses $\psi \subseteq \Phi$. Solving the MaxSAT problem consists in finding an assignment which maximizes the number of satisfied clauses for a given CNF formula.

Definition 1 (Max-resolution [6,8]). The inference rule for Max-SAT, maxresolution, is defined as follows:

$$
\frac{c=\left\{x, y_{1}, \ldots, y_{s}\right\}, c^{\prime}=\left\{\bar{x}, z_{1}, \ldots, z_{t}\right\}}{c r=\left\{y_{1}, \ldots, y_{s}, z_{1}, \ldots, z_{t}\right\}, c c_{1}, \ldots, c c_{t}, c c_{t+1}, \ldots, c c_{t+s}}
$$

where the compensation clauses are defined as follows:

$$
\begin{gathered}
c c_{1}=\left\{x, y_{1}, \ldots, y_{s}, \overline{z_{1}}\right\} \\
c c_{2}=\left\{x, y_{1}, \ldots, y_{s}, z_{1}, \overline{z_{2}}\right\} \\
\ldots \\
c c_{t}=\left\{x, y_{1}, \ldots, y_{s}, z_{1}, \ldots, z_{t-1}, \overline{z_{t}}\right\} \\
c c_{t+1}=\left\{\bar{x}, z_{1}, \ldots, z_{t}, \overline{y_{1}}\right\} \\
c c_{t+2}=\left\{\bar{x}, z_{1}, \ldots, z_{t}, y_{1}, \overline{y_{2}}\right\} \\
\ldots \\
c c_{t+s}=\left\{\bar{x}, z_{1}, \ldots, z_{t}, y_{1}, \ldots, y_{2}, \overline{y_{s}}\right\}
\end{gathered}
$$


Unlike the SAT inference rule, max-resolution replaces the premises in the rule by its conclusions. Furthermore, it produces an equivalent formula, i.e., it preserves the number of unsatisfied clauses for any assignment. The results established in this paper can be easily extended to weighted Max-SAT formulas (hard clauses can be included with infinite weights in the case of partial formulas) using the weighted version of max-resolution introduced in [6].

Notation. Let $\psi$ be an IS of a CNF formula $\Phi$ and $S=\left\langle x_{1}, \ldots, x_{k}\right\rangle$ be a sequence of variables appearing in $\psi$. We denote $\Theta(\psi, S)$ the set of clauses obtained from $\psi$ after the application of max-resolution steps in accordance to the sequence $S$, i.e., $\Theta(\psi, S)=\theta\left(\theta \ldots\left(\theta\left(\psi, x_{1}\right), x_{2}\right) \ldots, x_{k}\right)$ where $\theta(\psi, x)$ denotes the application of the max-resolution step defined above on two clauses $c$ and $c^{\prime}$ such that $x \in c$ and $\bar{x} \in c^{\prime}$.

Next, we recall the notion of UP-resilience [4]. The empirical study conducted in [2] shows a correlation between the decrease of the number of propagations, the decrease of the number of detected ISs and the increase of the number of decisions, i.e., if the number of propagations is reduced, then less ISs will be detected and the quality of the LB estimation will be reduced. This observation was stated more clearly in [4] as the fragmentation phenomenon which was the main motivation behind the introduction of the UP-resilience property. This phenomenon, showcased in Example 1, occurs when clauses are fragmented into two (or more) clauses after transformation by max-resolution which may obstruct their exploitation by the SUP mechanism.

Example 1. we consider the IS $\psi=\left\{\left\{x_{1}\right\},\left\{x_{2}\right\},\left\{x_{3}\right\},\left\{\overline{x_{3}}, \overline{x_{4}}\right\},\left\{\overline{x_{1}}, \overline{x_{2}}, x_{4}\right\}\right\}$ detected by the sequence of unit propagations represented in the form of an implication graph [15] in Fig.1. The max-resolution transformation of this IS with respect to the variable sequence $S=\left\langle x_{4}, x_{3}, x_{2}, x_{1}\right\rangle$ (in the reverse order of propagation) is given on the right in Fig.1.
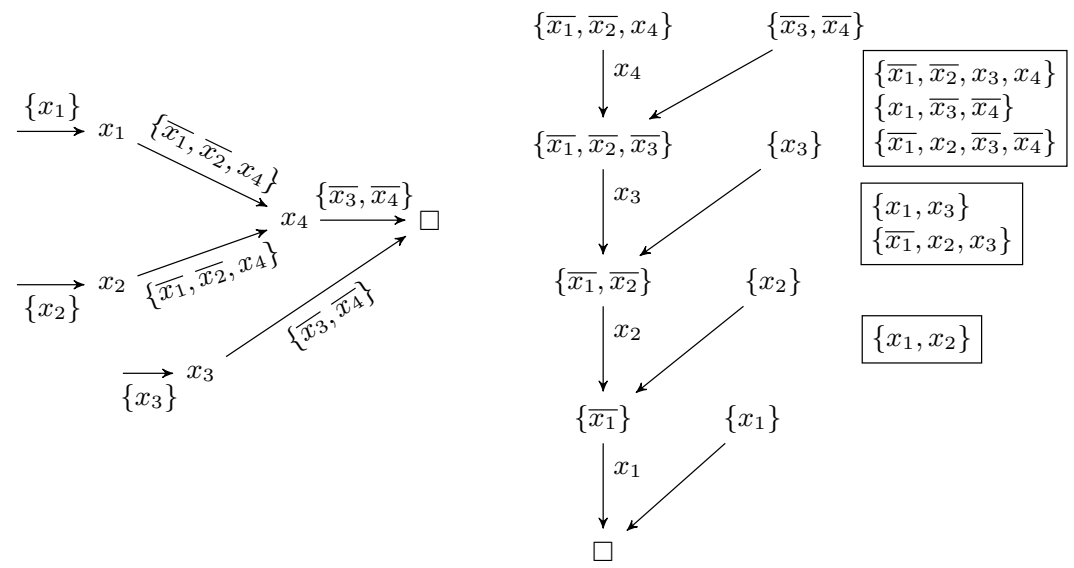

Fig. 1. Implication graph corresponding to a propagation sequence of $\psi$ in example 1 and its transformation by max-resolution, where compensation clauses for each step are represented in boxes. 
If the unique neighbor of $x_{1}$ in the implication graph is set to true in the transformed IS, we obtain $\left.\Theta(\psi, S)\right|_{\left\{x_{4}\right\}}=\left\{\left\{x_{1}, \overline{x_{3}}\right\},\left\{x_{1}, x_{3}\right\},\left\{x_{1}, x_{2}\right\},\left\{\overline{x_{1}}, x_{2}, \overline{x_{3}}\right\}\right.$, $\left.\left\{\overline{x_{1}}, x_{2}, x_{3}\right\}\right\}$. Clearly, the literal $x_{1}$ can't be propagated in $\left.\Theta(\psi, S)\right|_{\left\{x_{4}\right\}}$. We can produce the resolvent $x_{1}$ if we perform a max-resolution step between the clauses $\left\{x_{1}, x_{3}\right\}$ and $\left\{x_{1}, \overline{x_{3}}\right\}$ but the SUP mechanism alone cannot ensure the propagation of this literal in the transformed IS even with respect to its neighborhood in the implication graph. We say that the information leading to the propagation of $x_{1}$ was fragmented into several compensation clauses.

As an IS can be detected by different propagation sequences each corresponding to an implication graph [15]. So, before recalling the definition of the UP-resilience property, we give the formal definition of an implication graph of an IS in the context of $\mathrm{BnB}$ solvers for MaxSAT and of possible neighborhoods of a literal appearing in an IS.

Definition 2 (Implication graph of an IS). Let $\Psi$ be an IS of a CNF formula $\Phi$ and $I$ an assignment. We suppose that exactly one clause is falsified by I (SUP stopped when the first empty clause is generated). An implication graph of $\Psi$ is a directed acyclic graph $G=(V, A)$ defined as follows :

$-V=\{l \in I\} \cup\left\{\diamond_{c} \mid c \in \Psi\right.$ and $\left.|c|=1\right\} \cup\{\square\}$

- $A=\left\{\left(l, l^{\prime}, c\right) \mid l, l^{\prime} \in I\right.$ and $c \in \Psi$ is reduced by $l$ and propagates $\left.l^{\prime}\right\} \bigcup$ $\left\{\left(\diamond_{c}, l, c\right) \mid l \in I\right.$ and $\left.c=\{l\} \in \Psi\right\} \bigcup$

$\{(l, \square, c) \mid l \in I$ and $c \in \Psi$ is falsified by $I$ and $\bar{l} \in \Psi\}$

The directed edges are labeled by clauses and the nodes $\diamond$ are omitted in $G$.

Definition 3 (Possible neighborhoods [4]). Let $\phi$ be a CNF formula and $\psi$ an IS. For a literal $l$ appearing in $\psi$, we define its possible neighborhoods as pneigh $(l)=\left\{\right.$ neigh $_{G}(l) \mid G=(V, A)$ implication graph of $\psi$ s.t. $\left.l \in V\right\}$ where $n^{n e i g h_{G}}(l)$ denotes the neighbors of $l$ in $G$. We extend this definition on any set of literals $L$ appearing in $\psi$ as pneigh $(L)=\left\{\bigcup_{l \in L}\right.$ neigh $_{G}(l) \mid G=(V, A)$ implication graph of $\psi$ s.t. $L \subseteq V\}$.

Definition 4 (UP-resilience [4]). Let $\phi$ be a CNF formula, $\psi$ an IS and $S$ a sequence of variables appearing in $\psi$. The transformation $\Theta(\psi, S)$ is UP-resilient for a literal $l$ appearing in $\psi$ iff $\forall N \in$ pneigh $_{\psi}(l): \square \in N$ or $l$ can be propagated in $\left.\Theta(\psi, S)\right|_{N}$ where $\left.\Theta(\psi, S)\right|_{N}$ denotes the set of clauses in $\Theta(\psi, S)$ with the literals appearing in $N$ set to true. We say that $\Theta(\psi, S)$ is UP-resilient if it is UP-resilient for all the literals appearing in $\psi$.

We finish this section by a brief overview of UCS patterns which were introduced and empirically studied in order to extend the learning mechanisms in BnB Max-SAT solvers [2].

Definition 5 (Unit Clause Subset [2]). Let $\phi$ be a CNF formula and $k \geq 2$. $A$ k-Unit Clause Subset, denoted $k$-UCS, is a set of clauses $\left\{c_{1}, \ldots, c_{k}\right\} \subseteq \phi$ such that there exists a sequence of max-resolution steps on $c_{1}, \ldots, c_{k}$ that produces a unit clause resolvent. In particular, if $\forall i \in\{1, \ldots, k\}$ we have $\left|c_{i}\right|=2$, it is a binary $k-U C S$, denoted $k^{b}-U C S$. 
Example 2. The following patterns:

$$
\frac{\left\{l_{1}, l_{2}\right\},\left\{l_{1}, \overline{l_{2}}\right\}}{\left\{l_{1}\right\}}\left(P_{1}\right) \frac{\left\{l_{1}, l_{2}\right\},\left\{l_{1}, l_{3}\right\},\left\{\overline{l_{2}}, \overline{l_{3}}\right\}}{\left\{l_{1}\right\},\left\{l_{1}, l_{2}, l_{3}\right\},\left\{\overline{l_{1}}, \overline{l_{2}}, \overline{l_{3}}\right\}}\left(P_{2}\right)
$$

which are learned in state of the art BnB solvers, correspond respectively to a $2^{b}$-UCS and a $3^{b}$-UCS.

Definition 6 (First Unique Implication Point [15] ). Let $G$ be an implication graph. A Unique Implication Point (UIP) is any node in $G$ such that any path from the literals propagated by unit clauses to the conflict node must pass through it. The First UIP (FUIP) is the UIP closest to the conflict node.

It is important to note that UCS patterns have a high apparition frequency (in more than $57 \%$ of the detected ISs [2]). Furthermore, certain $k$-UCS patterns are easily detectable by analyzing the implication graph of the obtained IS [2]. Indeed, the clauses which are between the conflict and the FUIP produce a unit resolvent clause if they are transformed by max-resolution in the reverse propagation order. From here on, we will focus on such $k$-UCS patterns.

\section{Preliminaries and Motivation}

In this section, we explain how the notion of UP-resilience quantifies the impact of max-resolution on the SUP mechanism and thus on the detection of Inconsistent Subsets. This is highlighted in Property 1 which proves that UP-resilient transformations maintain the propagations which are not necessary anymore to an inconsistent subset. We provide a different proof for this property that is shorter and simpler than the one in [4]. We also show, in Propositions 1 and 2 , that the transformations corresponding to patterns $\left(P_{1}\right)$ and $\left(P_{2}\right)$ are UPresilient which contributes to explain from a theoretical point of view the empirical efficiency of these patterns. We give detailed proofs of these propositions to emphasize the fact that they are valid for any possible order of application of max-resolution, a fact that will be of importance in the discussion of our results in Section 4.

Property 1. Let $\phi$ be a CNF formula, $\psi$ an IS of $\phi$ and $S$ a sequence of variables appearing in $\psi$. For any set of literals $L$ appearing in $\psi$, if the transformation $\Theta(\psi, S)$ is UP-resilient for $L$ then $\forall N \in$ pneigh $(L): \square \in N$ or every literal $l \in L$ can be propagated in $\left.\Theta(\psi, S)\right|_{N \backslash\{l\}}$.

Proof. We prove this property by induction on $|L|=n$ :

- If $n=1$ then $L=\{l\}$ and the property is verified.

- Suppose the property is true for every set of size $n$. Let $L$ be of size $n+1$ and $l$ a literal in $L$. We set $L^{\prime}=L \backslash\{l\}$ and let $N \in \operatorname{pneigh}(L)$. Clearly, $N=N_{1} \cup N_{2}$ where $N_{1} \in$ pneigh $\left(L^{\prime}\right)$ and $N_{2} \in$ pneigh $(l)$. Moreover, since $\left|L^{\prime}\right|=n$, we know by induction that $\forall N \in \operatorname{pneigh}\left(L^{\prime}\right): \square \in N$ or every 
literal $l^{\prime}$ in $L^{\prime}$ can be propagated in $\left.\Theta(\psi, S)\right|_{N \backslash\left\{l^{\prime}\right\}}$. In particular, $\square \in N_{1}$ or every literal $l^{\prime}$ in $L^{\prime}$ can be propagated in $\left.\Theta(\psi, S)\right|_{N_{1} \backslash\left\{l^{\prime}\right\}}$. Also, The transformation $\Theta(\psi, S)$ is UP-resilient for $L$ and particularly for $l$ and thus, we have $\forall N \in$ pneigh $(l): \square \in N$ or $l$ can be propagated in $\left.\Theta(\psi, S)\right|_{N}$. In particular, $\square \in N_{2}$ or $l$ can be propagated in $\left.\Theta(\psi, S)\right|_{N_{2}}$. Thus, We have the following cases:

- If $\square \in N_{1}$ or $\square \in N_{2}$ then $\square \in N$

- Else every literal $l^{\prime}$ in $L^{\prime}$ and $l$ can be propagated respectively in $\left.\Theta(\psi, S)\right|_{N_{1} \backslash\left\{l^{\prime}\right\}}$ and $\left.\Theta(\psi, S)\right|_{N_{2}}$. Therefore, the clauses that ensure the propagation of every literal $l^{\prime}$ in $L^{\prime}$ in $\left.\Theta(\psi, S)\right|_{N_{1} \backslash\left\{l^{\prime}\right\}}$ also ensure their propagation in $\left.\Theta(\psi, S)\right|_{\left(N_{1} \cup N_{2}\right) \backslash\left\{l^{\prime}\right\}}$ and, similarly, the clauses that ensure the propagation of 1 in $\left.\Theta(\psi, S)\right|_{N_{2}}$ also ensure its propagation in $\left.\Theta(\psi, S)\right|_{\left(N_{1} \cup N_{2}\right) \backslash\{l\}}$.

We deduce that $\forall N \in$ pneigh $(L): \square \in N$ or every literal $l$ in $L$ can be propagated in $\left.\Theta(\psi, S)\right|_{N \backslash\{l\}}$.

Proposition 1. Let $\Phi$ be a CNF formula, $\Psi$ an $I S$ and $\Psi^{\prime} \subset \Psi$ such that $\Psi^{\prime}$ matches the premises of pattern $\left(P_{1}\right)$. Then, the max-resolution transformation described in $\left(P_{1}\right)$ is UP-resilient.

Proof. $\psi^{\prime}=\left\{\left\{l_{1}, l_{2}\right\},\left\{l_{1}, \overline{l_{2}}\right\}\right\}$. Therefore, there are two possible propagation sequences whose implication graphs are represented in Fig.2. Since all possible neighborhoods of literals $\overline{l_{1}}, l_{2}$ and $\overline{l_{2}}$ contain the empty clause, the transformation of $\psi^{\prime}$ as in $\left(P_{1}\right)$, with respect to the only possible variable sequence $S=\left\langle\operatorname{var}\left(l_{2}\right)\right\rangle$, is UP-resilient.
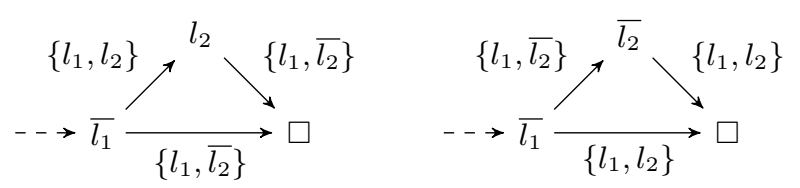

Fig. 2. Implication graphs corresponding to the possible propagation sequences for an IS containing the premises of pattern $\left(P_{1}\right)$.

Proposition 2. Let $\Phi$ be a $C N F$ formula, $\Psi$ an $I S$ and $\Psi^{\prime} \subset \Psi$ such that $\Psi^{\prime}$ matches the premises of pattern $\left(P_{2}\right)$. Then, the max-resolution transformation described in $\left(P_{2}\right)$ is UP-resilient.

Proof. $\psi^{\prime}=\left\{\left\{l_{1}, l_{2}\right\},\left\{l_{1}, l_{3}\right\},\left\{\overline{l_{2}}, \overline{l_{3}}\right\}\right\}$. Therefore, there are two possible propagation sequences whose implication graphs are represented in Fig.3. There are two max-resolution application orders $S_{1}=\left\langle\operatorname{var}\left(l_{2}\right), \operatorname{var}\left(l_{3}\right)\right\rangle$ and $\left.S_{2}=<\operatorname{var}\left(l_{3}\right), \operatorname{var}\left(l_{2}\right)\right\rangle$ that produce the same transformation described by pattern $\left(P_{2}\right)$. Since all possible neighborhoods of $l_{2}$ and $\overline{l_{2}}$ contain the empty clause, the transformation of $\psi$ by max-resolution is UP-resilient for $l_{2}$ and $\overline{l_{2}}$. We have pneigh $\left(\overline{l_{1}}\right)=\left\{\left\{l_{3}, \square\right\} \cup \operatorname{pred}\left(\overline{l_{1}}\right),\left\{l_{2}, l_{3}\right\} \cup \operatorname{pred}\left(\overline{l_{1}}\right)\right\}$, where $\left.\operatorname{pred}\left(\overline{l_{1}}\right)\right)$ 
denotes the predecessors of $\overline{l_{1}}$, and clearly the clause $c=\left\{\overline{l_{1}}, \overline{l_{2}}, \overline{l_{3}}\right\}$ propagates $\overline{l_{1}}$ when the literals $l_{2}, l_{3}$ in its second neighborhood are set to true. Also, pneigh $\left(l_{3}\right)=\left\{\left\{\overline{l_{1}}, \square\right\},\left\{\overline{l_{1}}, \overline{l_{2}}\right\}\right\}$ and similarly the clause $c^{\prime}=\left\{l_{1}, l_{2}, l_{3}\right\}$ propagates $l_{3}$ when the literals in its neighborhood $\left\{\overline{l_{1}}, \overline{l_{2}}\right\}$ are set to true.

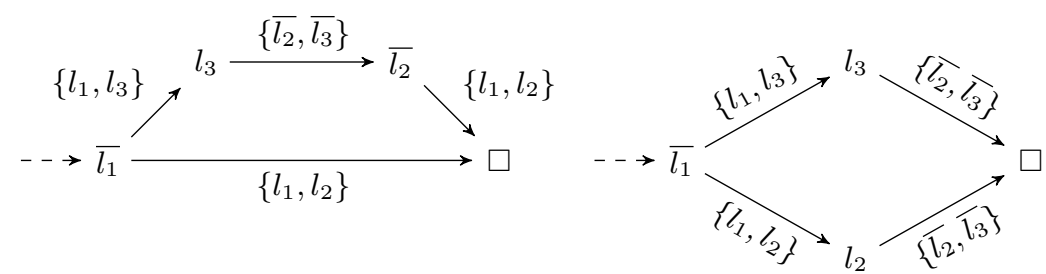

Fig. 3. Implication graphs corresponding to the possible propagation sequences for an IS containing the premises of pattern $\left(P_{2}\right)$.

Corollary 1. For $k \in\{2,3\}, k^{b}$-UCSs are UP-resilient.

Proof. $2^{b}$-UCSs and $3^{b}$-UCSs are all of the respective forms $\Psi_{2^{b}}=\left\{\left\{l_{1}, l_{2}\right\},\left\{l_{1}, \overline{l_{2}}\right\}\right\}$ and $\Psi_{3^{b}}=\left\{\left\{l_{1}, l_{2}\right\},\left\{l_{1}, l_{3}\right\},\left\{\overline{l_{2}}, \overline{l_{3}}\right\}\right\}$ which correspond to the premises of patterns $\left(P_{1}\right)$ and $\left(P_{2}\right)$. Thus, we obtain the wanted result using Propositions 1 and 2.

The previous results establish that UP-resilient transformations can't negatively impact the SUP mechanism and that the transformations learned in state of the art BnB solvers for Max-SAT in the form of patterns $\left(P_{1}\right)$ and $\left(P_{2}\right)$ are UP-resilient. One major challenge is to use this property to help decide the relevance of application of max-resolution transformations either by devising an efficient algorithm to verify the property on potential transformations or by using it to characterize the transformation of certain patterns. Since checking the property on potential transformations seems computationally costly, we tackle in the next section the second problem by generalizing the result of Corollary 1.

\section{Contributions}

In this section, we prove that binary $k$-UCSs are UP-resilient by providing two different orders that ensure the UP-resilience of their transformation by maxresolution. We also show that unlike the given orders, the current used mechanisms can't ensure UP-resilience for these patterns which provides an explanation to the empirical results in [2] and shows that our results can help extend the current used patterns in state of the art solvers. Furthermore, we generalize our result on the resilience of $k^{b}$-UCSs to $k$-UCSs where all clauses are binary except one of any size that is involved in the conflict. We start by proving the following lemma in order to characterize the detected implication graphs of such $k$-UCSs.

Lemma 1. Let $k \geq 2$ and $\psi$ be a $k$-UCS whose clauses are binary except for the conflict clause of size $s \geq 2$, recognized by the FUIP $l$ in an implication graph $G$ of an IS such that $|\operatorname{succ}(l)|=s$. Then, there exists exactly $s$ disjoint paths from $l$ to $\square$ in $G$. 
Proof. Since $l$ is a UIP, all the paths from the literals propagated by unit clauses to the conflict node in $G$ pass through it. We have $|\operatorname{succ}(l)|=s$. Therefore, there are at least $s$ different paths from $l$ to $\square$ in $G$. Let $p_{1}, \ldots, p_{s}$ be those paths. Suppose we have a different path $p_{s+1}$ from $l$ to $\square$. We have two possible cases:

$-|\operatorname{pred}(\square)| \neq s$. This is absurd since the conflict clause $c$ is of size $s$ and thus $|\operatorname{pred}(\square)|=s$.

- Else, since $|\operatorname{pred}(\square)|=s$, there exists $l^{\prime} \neq l \in p_{s+1}$ and $i \in\{1, \ldots, s\}$ such that $l^{\prime} \in p_{i}$ and $\left|\operatorname{pred}\left(l^{\prime}\right)\right|>1$. This is absurd since all clauses of the $k$-UCS except $c$ are binary.

We deduce that there are exactly $s$ different paths from $l$ to $\square$ in $G$. The same argument of the second case ensures that these paths are disjoint.

As explained in Section 2, when a UCS is detected, we know that the reverse propagation order ensures the production of a unit clause after the transformation but, in general, this is not necessarily true for all the orders. Since this is the main feature of UCS patterns, we must ensure that the introduced orders produce unit clauses. It is important to note that the condition on the successors of the FUIP in Lemma 1 ensures this property for all possible orders. We start by proving the UP-resilience of $k^{b}$-UCSs. To this end, we show in the next proposition that the condition on the FUIP successors in Lemma 1 is always verified for $k^{b}$-UCSs. Later, when we generalize our result, we only consider the graphs described by Lemma 1, i.e., which verify the condition on the successors of the FUIP.

Proposition 3. Let $k \geq 2$ and $\psi$ be a $k^{b}$-UCS recognized by the FUIP $l$ in an implication graph $G$ of an IS. Then, $|\operatorname{succ}(l)|=2$.

Proof. Suppose that $|\operatorname{succ}(l)| \neq 2$. We have two possible cases:

- if $|\operatorname{succ}(l)|>2$ then, since $|\operatorname{succ}(\square)|=2$, there exists a literal with two predecessors. This is absurd since all the clauses are binary.

- if $|\operatorname{succ}(l)|=1$ then $l$ is not the FUIP which is absurd.

Definition 7 (Path Resolvent Order). Let $p_{1}=\left\langle l, l_{1}^{p_{1}}, \ldots, l_{n_{1}}^{p_{1}}, \square\right\rangle\left(n_{1} \geq 0\right)$ and $p_{2}=\left\langle l, l_{1}^{p_{2}}, \ldots, l_{n_{2}}^{p_{2}}, \square\right\rangle\left(n_{2} \geq 0\right)$ denote two disjoint paths from $l$ to $\square$. The Path Resolvent Order (PRO) of $p_{1}$ and $p_{2}$ is defined as $\operatorname{PRO}\left(p_{1}, p_{2}\right)=$ $\left\langle\operatorname{var}\left(l_{1}^{p_{1}}\right), \ldots, \operatorname{var}\left(l_{n_{1}}^{p_{1}}\right), \operatorname{var}\left(l_{1}^{p_{2}}\right), \ldots, \operatorname{var}\left(l_{n_{2}}^{p_{2}}\right)\right\rangle$.

Theorem 1. For any $k \geq 2$, the transformation of $k^{b}$-UCSs with respect to PRO is UP-resilient.

Proof. Let $k \geq 2$ and $\psi$ be a $k^{b}$-UCS recognized by the FUIP $l$ in the implication graph $G$ of an IS. By Lemma 1 and Proposition 3, we know that there are 2 disjoint paths from $l$ to $\square$ in G. Let $p_{1}=\left\langle l, l_{1}^{p_{1}}, \ldots, l_{n_{1}}^{p_{1}}, \square\left\langle\left(n_{1} \geq 0\right)\right.\right.$ and $p_{2}=$ $\left\langle l, l_{1}^{p_{2}}, \ldots, l_{n_{2}}^{p_{2}}, \square\right\rangle\left(n_{2} \geq 0\right)$ denote these paths in $G$ where $n_{1}+n_{2}=k-1$. And, 
suppose w.l.o.g that $l_{n_{1}}^{p_{1}}=l^{\prime}$ is the conflict literal, i.e., the last propagated literal. We have two possible propagation sequences whose implication graphs are $G$ and $G^{\prime}$ represented in Fig.4.
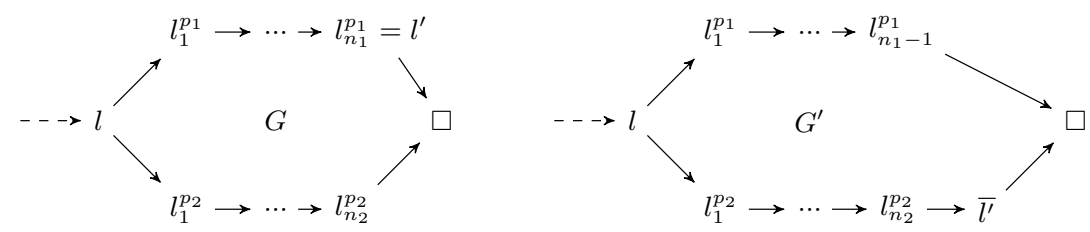

Fig. 4. Implication graphs corresponding to the possible propagation sequences for $k^{b}$-UCSs.

We prove that the max-resolution transformation relatively to the order $O=$ $P R O\left(p_{1}, p_{2}\right)$ is UP-resilient:

- The clause propagating $l$ is not deleted after the transformation by maxresolution relatively to the order $O$ so it clearly propagates $l$ if its predecessors are set to true and thus the transformation by max-resolution relatively to the order $O$ is UP-resilient for $l$. This argument also applies for the literals that were involved in the propagation of $l$.

- All possible neighborhoods of literals $l_{n_{1}}^{p_{1}}=l^{\prime}$ and $\overline{l^{\prime}}$ contain the empty clause. Therefore, the transformation by max-resolution relatively to the order $O$ is UP-resilient for $l^{\prime}$ and $\overline{l^{\prime}}$.

- For $i \in\{1,2\}$, we set $l_{0}^{p_{i}}=l$. Every literal $l_{j}^{p_{i}}$ such that $1 \leq j<n_{i}$ admits exactly one neighborhood neigh $\left(l_{j}^{p_{1}}\right)=\left\{l_{j-1}^{p_{i}}, l_{j+1}^{p_{i}}\right\}$ that doesn't contain the empty clause. Similarly, for $l_{n_{2}}^{p_{2}}$ we have neigh $\left(l_{n_{2}}^{p_{2}}\right)=\left\{l_{n_{2}-1}^{p_{i}}, \overline{l^{\prime}}\right\}$. The maxresolution step on $\operatorname{var}\left(l_{j}^{p_{1}}\right)\left(1 \leq j<n_{i}\right)$ is of the form:

$$
\frac{\left\{\bar{l}, l_{j}^{p_{i}}\right\},\left\{\overline{l_{j}^{p_{i}}}, l_{j+1}^{p_{i}}\right\}}{\left\{\bar{l}, l_{j+1}^{p_{i}}\right\},\left\{\bar{l}, l_{j}^{p_{i}}, \overline{l_{j+1}^{p_{i}}}\right\},\left\{l, \overline{p_{j}^{p_{i}}}, l_{j+1}^{p_{i}}\right\}}
$$

The clause $c=\left\{l, \overline{l_{j}^{p_{i}}}, l_{j+1}^{p_{i}}\right\}$ clearly ensures the propagation of literal $l_{j+1}^{p_{i}}$ if $l_{j}^{p_{i}} \in \operatorname{neigh}\left(l_{j+1}^{p_{i}}\right)$ is set to true since $\bar{l}$ is propagated by the unit resolvent clause $\{\bar{l}\}$. Also, for $j=1$, the clause $c^{\prime}=\left\{\bar{l}, l_{1}^{p_{1}}, \overline{l_{2}^{p_{1}}}\right\}$ ensures the propagation of $l_{1}^{p_{1}}$ if $l, l_{2}^{p_{1}} \in$ neigh $\left(l_{1}^{p_{1}}\right)$ are set to true. Thus, We deduce that the transformation is UP-resilient for $l_{j}^{p_{i}}$ where $1 \leq j \leq n_{i}\left(j \neq n_{1}\right)$.

We conclude that the transformation of $\psi$ by max-resolution relatively to the order $O$ is UP-resilient.

Definition 8 (Path Resolvent Circular Order). Let $p_{1}=\left\langle l, l_{1}^{p_{1}}, \ldots, l_{n_{1}}^{p_{1}}, \square\right\rangle$ $\left(n_{1} \geq 0\right)$ and $p_{2}=\left\langle l, l_{1}^{p_{2}}, \ldots, l_{n_{2}}^{p_{2}}, \square\right\rangle\left(n_{2} \geq 0\right)$ denote two disjoint paths from $l$ to $\square$. The Path Resolvent Circular Order (PRCO) of $p_{1}$ and $p_{2}$ is defined as $P R C O\left(p_{1}, p_{2}\right)=\left\langle\operatorname{var}\left(l_{1}^{p_{1}}\right), \ldots, \operatorname{var}\left(l_{n_{1}}^{p_{1}}\right), \operatorname{var}\left(l_{n_{2}}^{p_{2}}\right), \ldots, \operatorname{var}\left(l_{1}^{p_{2}}\right)\right\rangle$. 
Theorem 2. For any $k \geq 2$, the transformation of $k^{b}$-UCSs with respect to PRCO is UP-resilient.

Proof. Let $k \geq 2$ and $\psi$ be a $k^{b}$-UCS recognized by the FUIP $l$ in the implication graph $G$ of an IS. By Lemma 1 and Proposition 3 , let $p_{1}=\left\langle l, l_{1}^{p_{1}}, \ldots, l_{n_{1}}^{p_{1}}, \square\right\rangle\left(n_{1} \geq\right.$ $0)$ and $p_{2}=\left\langle l, l_{1}^{p_{2}}, \ldots, l_{n_{2}}^{p_{2}}, \square\right\rangle\left(n_{2} \geq 0\right)$ denote the two disjoint paths from $l$ to $\square$ in $G$ where $n_{1}+n_{2}=k-1$. And, suppose w.l.o.g that $l_{n_{1}}^{p_{1}}=l^{\prime}$ is the conflict literal. We have two possible propagation sequences whose implication graphs are $G$ and $G^{\prime}$ represented in Fig.4. We prove that the max-resolution transformation relatively to the order $O=P R C O\left(p_{1}, p_{2}\right)$ is UP-resilient:

- The same arguments in the proof of Theorem 1 ensure the UP-resilience of the transformation respectively to $O$ for $l_{j}^{p_{1}}\left(1 \leq j \leq n_{1}\right)$ and $\overline{l^{\prime}}$ as well as $l$ and all the literals involved in its propagation.

- Every literal $l_{j}^{p_{2}}$ such that $1 \leq j \leq n_{2}$ admits exactly one neighborhood neigh $\left(l_{j}^{p_{2}}\right)=\left\{l_{j-1}^{p_{2}}, l_{j+1}^{p_{2}}\right\}$ that doesn't contain the empty clause (we set $l_{0}^{p_{2}}=$ $l$ and $\left.l_{n_{2}+1}^{p_{2}}=\overline{l^{\prime}}\right)$. The max-resolution step on $\operatorname{var}\left(l_{j}^{p_{2}}\right)(j \neq 1)$ is of the form :

$$
\frac{\left\{\bar{l}, \overline{l_{j}^{p_{2}}}\right\},\left\{l_{j}^{p_{2}}, \overline{l_{j-1}^{p_{2}}}\right\}}{\left\{\bar{l}, \overline{l_{j-1}^{p_{2}}}\right\},\left\{\bar{l}, \overline{l_{j}^{p_{2}}}, l_{j-1}^{p_{2}}\right\},\left\{l, l_{j}^{p_{2}}, \overline{l_{j-1}^{p_{2}}}\right\}}
$$

The clause $c=\left\{l, l_{j}^{p_{2}}, \overline{l_{j-1}^{p_{2}}}\right\}$ clearly ensures the propagation of literal $l_{j}^{p_{2}}$ when $l_{j-1}^{p_{2}} \in \operatorname{neigh}\left(l_{j}^{p_{2}}\right)$ is set to true since $\bar{l}$ is propagated by the unit resolvent clause $\{\bar{l}\}$. Also, the clause $c^{\prime}=\left\{\bar{l}, \overline{l_{2}^{p_{2}}}, l_{1}^{p_{2}}\right\}$, generated by the max-resolution step on $\operatorname{var}\left(l_{2}^{p_{2}}\right)$, clearly ensures the propagation of $l_{1}^{p_{2}}$ when its neighbors $l, l_{2}^{p_{2}} \in \operatorname{neigh}\left(l_{1}^{p_{2}}\right)$ are set to true. Thus, the transformation is UP-resilient for $l_{j}^{p_{2}}$ where $1 \leq j \leq n_{2}$.

We conclude that the transformation by max-resolution relatively to the order $O$ is UP-resilient.

There is a major difference between the orders we introduced. Indeed, PRCO ensures a linear input resolution transformation, i.e., at each intermediary maxresolution step we use the resolvent obtained in the previous step and a clause from the detected $k^{b}$-UCS. This is not always the case for PRO. The following result is an immediate consequence of either Theorem 1 or 2 .

Corollary 2. For any $k \geq 2$, there exists a UP-resilient transformation of $k^{b}$ UCSs.

Empirical results show that $2^{b}$-UCSs and $3^{b}$-UCSs, which correspond respectively to the patterns $(P 1)$ and $(P 2)$ have a positive impact on the performance of BnB solvers for Max-SAT $[2,10]$. The result in corollary 1 obtained through properties 1 and 2 prove that $2^{b}$-UCSs and $3^{b}$-UCSs are UP-resilient for any given order of application of max-resolution which explains why learning them 
has a positive impact regardless of the chosen order. This is not the case for $k^{b}$ UCSs when $k>3$. Empirical studies on the AHMAXSAT solver in [2] show that learning $4^{b}$-UCSs and $5^{b}$-UCSs had a major negative impact on its performance. This can be explained by the inadequacy of the max-resolution application orders used in state of the art BnB solvers for $k^{b}$-UCSs when $k>3$. Indeed, it was shown in [4] that the order impacts the UP-resilience of the transformations by comparing the following heuristics:

- Reverse Propagation Order (RPO) which applies max-resolution steps in the reverse order of propagation.

- Smallest Intermediary Resolvent (SIR) which applies the max-resolution steps based on the size of the resolvents between clauses, favoring the smallest ones [1].

In particular, the results show that the average percentage of UP-resilience of the transformations is comparatively higher with SIR. In the case of $k^{b}$-UCSs, these orders don't always ensure the UP-resilience property on the transformations. More specifically, the SIR heuristic becomes unusable since all the intermediary resolvents have the same size (binary) as shown in the proofs of Theorems 1 and 2, whereas the Reverse Propagation Order doesn't always ensure the UPresilience of the transformation as shown in the following example on a $4^{b}$-UCS which can be easily extended to any $k^{b}$-UCS for $k>4$.

Example 3. We consider the IS $\psi=\left\{\{l\},\left\{\bar{l}, l_{1}\right\},\left\{\bar{l}, l_{2}\right\},\left\{\overline{l_{1}}, l_{3}\right\},\left\{\overline{l_{2}}, \overline{l_{3}}\right\}\right\}$ detected by one of the possible implication graphs represented on the left in Fig.5 after the respective propagation of literals $l_{1}, l_{2}$ and $l_{3}$ (or $\overline{l_{3}}$ ). Clearly, the subset $\psi^{\prime}=\left\{\left\{\bar{l}, l_{1}\right\},\left\{\bar{l}, l_{2}\right\},\left\{\overline{l_{1}}, l_{3}\right\},\left\{\overline{l_{2}}, \overline{l_{3}}\right\}\right\} \subset \psi$ is a $4^{b}$-UCS recognized by the FUIP $l$. The max-resolution transformation of $\psi^{\prime}$ with respect to RPO which corresponds to the variable sequence $S=\left\langle\operatorname{var}\left(l_{3}\right), \operatorname{var}\left(l_{2}\right), \operatorname{var}\left(l_{1}\right)\right\rangle$ is represented on the right in Fig.5.
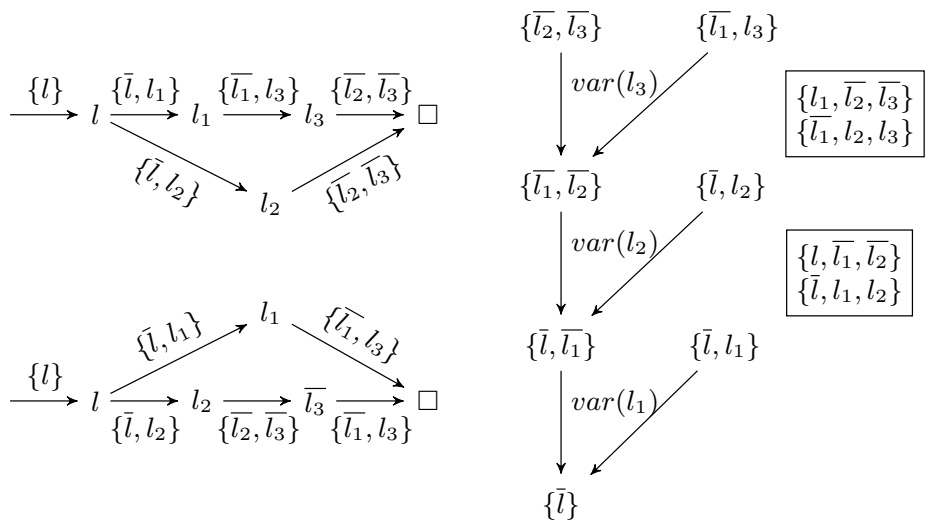

Fig. 5. Implication graphs corresponding to the possible propagation sequences of $\psi$ in Example 3 and the application of max-resolution steps relatively to RPO 
The literal $l_{1}$ has one neighborhood neigh $\left(l_{1}\right)=\left\{l, l_{3}\right\}$ that doesn't contain the empty clause. Clearly, the literal $l_{1}$ can't be propagated in $\left.\Theta(\psi, S)\right|_{n e i g h\left(l_{1}\right)}=$ $\left\{\left\{l_{1}, \overline{l_{2}}\right\},\left\{l_{1}, l_{2}\right\}\right\}$. Similarly, the fragmentation phenomenon also occurs for $l_{2}$ and we conclude that the transformation of $\psi^{\prime}$ relatively to RPO is not UP-resilient.

Now, we want to generalize our result to $k$-UCSs where all clauses are binary except one of any size that is involved in the conflict when the implication graph corresponds to the description in Lemma 1. A clause involved in the conflict is either the falsified clause or contains the conflict literal, i.e., the last propagated literal. Unfortunately, although PRCO has the advantage of ensuring a linear input transformation, we couldn't generalize it to obtain the wanted result. Nevertheless, we managed to prove our result using a generalization of PRO to a multitude of paths.

Definition 9 (Multiple Path Resolvent Order). Let $s \geq 2$ and $p_{1}=$ $\left\langle l, l_{1}^{p_{1}}, \ldots, l_{n_{1}}^{p_{1}}, \square\right\rangle, \ldots, p_{s}=\left\langle l, l_{1}^{p_{2}}, \ldots, l_{n_{s}}^{p_{s}}, \square\right\rangle$ denote $s$ disjoint paths from $l$ to $\square$. The Multiple Path Resolvent Order (MPRO) of $p_{1}, \ldots, p_{s}$ is defined inductively on $s$ as follows:

- If $s=2, M P R O\left(p_{1}, p_{2}\right)=P R O\left(p_{1}, p_{2}\right)$

- Else $\operatorname{MPRO}\left(p_{1}, \ldots, p_{s}\right)=\operatorname{PRO}\left(\left\langle l, M P R O\left(p_{1}, \ldots, p_{s-1}\right), \square\right\rangle, p_{s}\right)$.

Theorem 3. Let $k \geq 2$ and $\psi$ be a $k$-UCS whose clauses are binary except for the conflict clause $c$ of size $|c|=s \geq 3$, recognized by the FUIP $l$ in the implication graph $G$ of an IS such that $|\operatorname{succ}(l)|=s$. The transformation of $\psi$ with respect to $M P R O$ is UP-resilient.

Proof. We suppose w.l.o.g that $c=\left\{\overline{l_{1}}, \ldots, \overline{l_{s}}\right\}$. By Lemma 1 , there are exactly $s$ disjoint paths $p_{1}=\left\langle l, l_{1}^{p_{1}}, \ldots, l_{n_{1}}^{p_{1}}, \square\right\rangle, \ldots, p_{s}=\left\langle l, l_{1}^{p_{s}}, \ldots, l_{n_{s}}^{p_{s}}, \square\right\rangle$ from $l$ to $\square$ in the implication graph $G$, represented in Fig.6, such that $\sum_{i=1}^{s} n_{i}=k-1$ and $l_{n_{i}}^{p_{i}}=l_{i}$ for $i \in\{1, \ldots, s\}$. Other than $G$, there are exactly $\left(\begin{array}{c}i=1 \\ s\end{array}\right)=s$ possible implication graphs all similar to the graph $G^{\prime}$ represented in Fig.6.

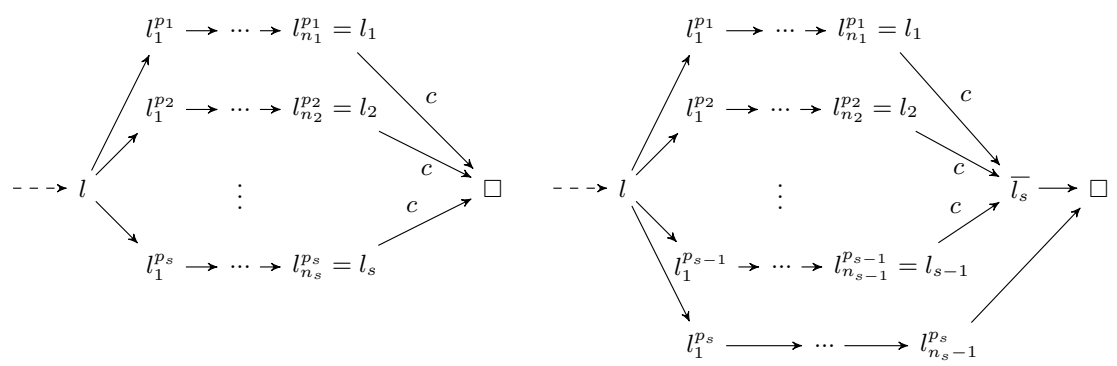

Fig. 6. Implication graphs corresponding to the possible propagation sequences for $k$-UCSs with binary clauses except for the conflict clause

We prove that the max-resolution transformation relatively to the order $O=$ $M P R O\left(p_{1}, \ldots, p_{s}\right)$ is UP-resilient: 
- The same arguments in the proof of Theorem 1 ensure the UP-resilience of the transformation respectively to $O$ for $l_{j}^{p_{i}}$ where $1 \leq i \leq s$ and $1 \leq j<n_{i}$ as well as $l$ and all the literals involved in its propagation. Furthermore, all the neighborhoods of literals $\overline{l_{1}}, \ldots, \overline{l_{s}}$ contain the empty clause.

- For $i \in\{1, \ldots, s\}, \forall N \in \operatorname{pneigh}\left(l_{n_{i}}^{p_{i}}\right)\left(n_{i}>1\right)$ s.t $\square \notin N$, we have $l_{n_{i}-1}^{p_{i}} \in N$ (exists since $n_{i}>1$ ). Clearly, the clause $c=\left\{l, \overline{l_{n_{i}-1}^{p_{i}}}, l_{n_{i}}^{p_{i}}\right\}$ obtained by the application of max-resolution on $\operatorname{var}\left(l_{n_{i}-1}^{p_{i}}\right)$ ensures the propagation of $l_{n_{i}}^{p_{i}}$ in any of these neighborhoods when $l_{n_{i}-1}^{p_{i}}$ is set to true since $\bar{l}$ is propagated by the unit resolvent clause $\{\bar{l}\}$. We deduce that the transformation relatively to the order O is UP-resilient for $l_{n_{i}}^{p_{i}}$ where $1 \leq i \leq s$ and $n_{i}>1$.

- We still need to prove the UP-resilience of the transformation for literals $l_{n_{i}}^{p_{i}}=l_{i}$ when $n_{i}=1$, with respect to their possible neighborhoods $\left\{l, \overline{l_{j}}\right\}$ for $j \in\{1, . ., s\} \backslash\{i\}$ not containing the empty clause. For this end, we prove by induction on $|c| \geq 3$ that the compensation clauses produced by the maxresolution steps on $\operatorname{var}\left(l_{1}\right), \ldots, \operatorname{var}\left(l_{s}\right)$ ensure the propagation of each literal $l_{i}$ if we consider the neighborhoods as mentioned above. For simplification, in the first max-resolution step, we replace $c$ by the clause $c^{\prime}=\left\{\bar{l}, \overline{l_{1}}, \ldots, \overline{l_{s}}\right\}$. This doesn't affect our result since we only omit a single clause containing the literal $l$ :

- If $|c|=3, c=\left\{\overline{l_{1}}, \overline{l_{2}}, \overline{l_{3}}\right\}$. The max-resolution steps are represented on the left in Fig.7 and we can easily check that the compensation clauses ensure the propagation of the literals $l_{i}$, for $1 \leq i \leq 3$, if we consider the neighborhoods mentioned above.

- Suppose the property is true for any clause of size $s \geq 3$. Let $c=$ $\left\{l_{1}, \ldots, l_{s+1}\right\}$ of size $s+1$. The first max-resolution step is represented on the right in Fig.7. The resolvent clause is $\left\{\bar{l}, l_{2}, \ldots, l_{s+1}\right\}$ and if we consider $c^{\prime}=\left\{l_{2}, \ldots, l_{s+1}\right\}$ of size $s$ we ensure by induction the propagation of any literal $l_{i}$ where $2 \leq i \leq s+1$ with respect to the neighborhoods $\left\{l, \overline{l_{j}}\right\}$ for $j \in\{2, . ., s+1\} \backslash\{i\}$. Thus, each compensation clause $c c_{k}=\left\{\bar{l}, l_{1}, \overline{l_{2}}, \ldots, \overline{l_{k}}, l_{k+1}\right\}$ for $k \in\{1, \ldots, s\}$ ensures the propagation of literal $l_{1}$ with respect to the neighborhood $\left\{l, \overline{l_{k+1}}\right\}$ since by induction the propagation of literals $l_{2}, \ldots, l_{k}$ is ensured in the same neighborhood. Now, we prove by induction on $k \in\{1, \ldots, s\}$ that the clause $c c_{k}$ ensures the propagation of $l_{k+1}$ with respect to the neighborhood $\left\{l, \overline{l_{1}}\right\}$ :

* If $k=1, c c_{1}=\left\{\bar{l}, l_{1}, l_{2}\right\}$ clearly ensures the propagation of $l_{2}$ with respect to the neighborhood $\left\{l, \overline{l_{1}}\right\}$.

* Suppose for $1 \leq k^{\prime}<k \leq s, c c_{k^{\prime}}$ ensures the propagation of $l_{k^{\prime}+1}$ with respect to the neighborhood $\left\{l, \overline{l_{1}}\right\} . c c_{k}=\left\{\bar{l}, l_{1}, \overline{l_{2}}, \ldots, \overline{l_{k}}, l_{k+1}\right\}$ clearly ensures the propagation of literal $l_{k+1}$ with respect to the neighborhood $\left\{l, \overline{l_{1}}\right\}$ since by induction the propagation of $l_{2}, \ldots, l_{k}$ is ensured in the same neighborhood by the clauses $c c_{1}, \ldots, c c_{k-1}$.

We conclude that the transformation by max-resolution relatively to the order $O$ is UP-resilient. 

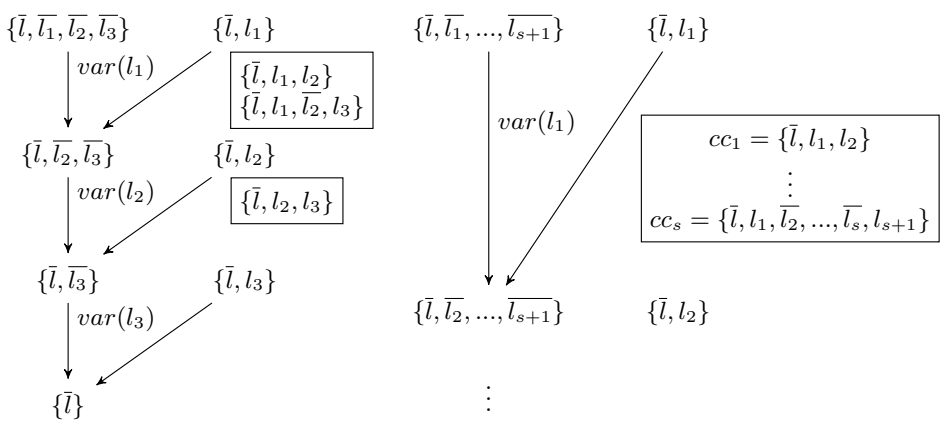

Fig. 7. Application of max-resolution steps on the variables of the non binary clause $c$ by induction on its size

Corollary 3. Let $k \geq 2$ and $\psi$ be a $k$-UCS whose clauses are binary except for a single clause $c$ of size $|c|=s \geq 3$ involved in the conflict, recognized by the FUIP $l$ in the implication graph $G$ of an IS such that $\operatorname{succ}(l)=s$. There exists a UP-resilient transformation of $\psi$.

Proof. If $c$ is the conflict clause then we obtain the result by Theorem 3. Else, $c$ contains the conflict literal and the detected implication graph $G$ has the same form as the second graph represented in Fig.6. Clearly, there is a propagation sequence where $c$ is falsified, i.e., corresponding to an implication graph $G^{\prime}$ similar to the first graph represented in Fig.6. Thus, we deduce the UP-resilience of the transformation with respect to MPRO through the same arguments in the proof of Theorem 3.

The SIR order is defined relatively to the size of the intermediary resolvents. Thus, it may theoretically simulate any order when the sizes of the resolvents are the same or many different orders when many resolvents share the same size which is the case of the studied UCSs. That's why this heuristic remains practically unusable even in the generalized case. Furthermore, RPO doesn't necessarily ensure the UP-resilience of $k$-UCSs described in the previous corollary. We finish this section by an example that highlights this fact. This example where the non binary clause is tertiary can be easily extended to any size $s>3$.

Example 4. We consider the IS $\psi=\left\{\{l\},\left\{\bar{l}, l_{1}\right\},\left\{\bar{l}, l_{2}\right\},\left\{\bar{l}, l_{3}\right\},\left\{\overline{l_{1}}, l_{4}\right\},\left\{\overline{l_{2}}, \overline{l_{3}}, \overline{l_{4}}\right\}\right\}$ (we name the tertiary clause c) detected by the first implication graph represented on the left in Fig. 8 after the respective propagation of literals $l_{1}, l_{2}, l_{3}$ and $l_{4}$. In the second graph on the left in the same figure, we represent another possible propagation sequence which outlines the possible neighborhood of $l_{4}$, neigh $\left(l_{4}\right)=\left\{l_{1}, \overline{l_{3}}\right\}$ not containing the empty clause. Clearly, the subset $\psi^{\prime}=\psi \backslash\{\{l\}\}$ is a 5-UCS recognized by the FUIP $l$ such that $c$ participates in the conflict and $|\operatorname{succ}(l)|=|c|=3$. The max-resolution transformation of $\psi^{\prime}$ with respect to RPO which corresponds to the variable sequence $S=$ $\left\langle\operatorname{var}\left(l_{4}\right), \operatorname{var}\left(l_{3}\right), \operatorname{var}\left(l_{2}\right), \operatorname{var}\left(l_{1}\right)\right\rangle$ is represented on the right in Fig.8. Clearly, the 
literal $l_{4}$ can't be propagated in $\left.\Theta(\psi, S)\right|_{\text {neigh }\left(l_{4}\right)}=\left\{\{\bar{l}\},\left\{\bar{l}, \overline{l_{2}}\right\},\left\{l_{2}, l_{4}\right\},\left\{\overline{l_{2}}, l_{4}\right\}\right\}$. We conclude that the transformation of $\psi^{\prime}$ relatively to RPO is not UP-resilient.

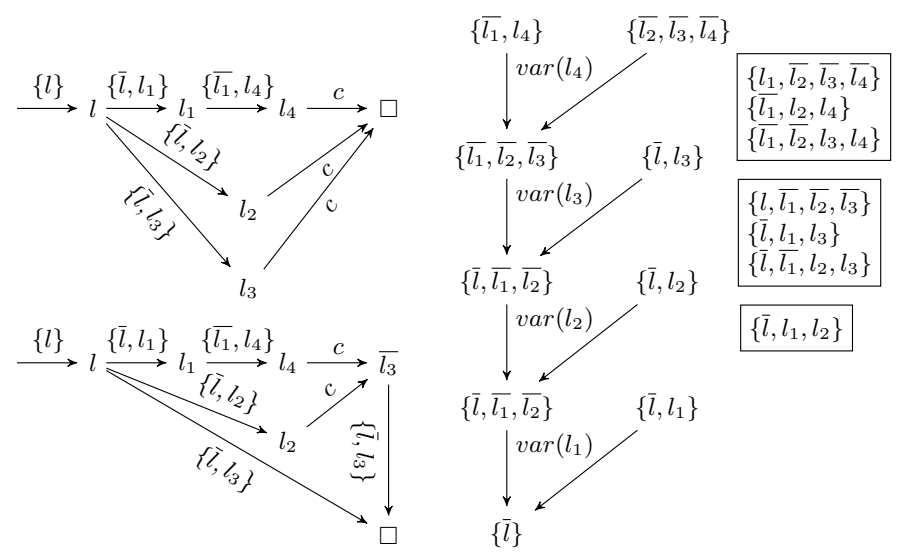

Fig. 8. Implication graphs corresponding to the possible propagation sequences of $\psi$ in Example 4 and the application of max-resolution steps relatively to RPO

\section{Conclusion}

In this paper, we proved that $k^{b}$-UCSs are UP-resilient with respect to two different orders PRO and PRCO. Then, we generalized this result to $k$-UCSs where all clauses are binary except one of any size involved in the conflict. We showed that unlike our orders, the current mechanisms don't necessarily ensure UP-resilience for these patterns. Thus, our orders can help extend the current patterns used in state of the art BnB solvers.

Until now, UP-resilience was mainly used to explain the impact of maxresolution transformations on the SUP mechanism. To our best knowledge, this is the first work in which this property is used to characterize the transformations by max-resolution in order to decide the relevance of their application. Indeed, this can be a starting point of a new approach to extend max-resolution patterns. In our case, we chose UCS patterns because they present several advantages: the introduction of unit clauses as well as the high frequency of their apparition. We also showed the limits of the current orders of application of max-resolution. In fact, this is the first work in which the proposed orders are introduced relatively to the structure of the implication graphs representing the possible propagation sequences of an IS.

The prospects of our research include the extension of our studies to $k$-UCSs in general. It also opens a new perspective for finding orders of application of max-resolution that ensure UP-resilience or maximizes its percentage by thoroughly studying the implication graphs corresponding to the propagation sequences of certain ISs. Finally, increasing knowledge about max-resolution can be useful for SAT-based solvers, which are mainly efficient on industrial instances, as some solvers, such as EVA [14], already exploit max-resolution to transform cores returned by SAT solvers. 


\section{References}

1. Abramé, A., Habet, D.: Efficient application of max-sat resolution on inconsistent subsets. In: O'Sullivan, B. (ed.) Principles and Practice of Constraint Programming. pp. 92-107. Springer International Publishing, Cham (2014)

2. Abramé, A., Habet, D.: On the extension of learning for Max-SAT. In: Endriss, U., Leite, J. (eds.) Proceedings of the 7th European Starting AI Researcher Symposium (STAIRS 2014). Frontiers in Artificial Intelligence and Applications, vol. 241, pp. 1-10. IOS Press (2014)

3. Abramé, A., Habet, D.: ahmaxsat: Description and Evaluation of a Branch and Bound Max-SAT Solver. Journal on Satisfiability, Boolean Modeling and Computation 9, 89-128 (2015)

4. Abramé, A., Habet, D.: On the resiliency of unit propagation to max-resolution. In: Yang, Q., Wooldridge, M. (eds.) Proceedings of the 24th International Joint Conference on Artificial Intelligence (IJCAI 2015). pp. 268-274. AAAI Press (2015)

5. Ansótegui, C., Bonet, M.L., Levy, J.: Solving (weighted) partial maxsat through satisfiability testing. In: Kullmann, O. (ed.) Theory and Applications of Satisfiability Testing - SAT 2009. pp. 427-440. Springer Berlin Heidelberg, Berlin, Heidelberg (2009)

6. Bonet, M.L., Levy, J., Manyà, F.: Resolution for max-sat. Artificial Intelligence 171(8), 606-618 (Jun 2007)

7. Davies, J., Bacchus, F.: Solving maxsat by solving a sequence of simpler sat instances. In: Lee, J. (ed.) International conference on principles and practice of constraint programming. pp. 225-239. Springer (2011)

8. Heras, F., Larrosa, J.: New inference rules for efficient Max-SAT solving. In: Cohn, A. (ed.) Proceedings of the 21st National Conference on Artificial Intelligence (AAAI 2006). pp. 68-73. AAAI Press (2006)

9. Küegel, A.: Improved exact solver for the weighted max-sat problem. In: Berre, D.L. (ed.) POS-10. Pragmatics of SAT. EPiC Series in Computing, vol. 8, pp. 15-27. EasyChair (2012)

10. Li, C.M., Manyà, F., Mohamedou, N.O., Planes, J.: Resolution-based lower bounds in maxsat. Constraints 15, 456-484 (Oct 2010)

11. Li, C.M., Manyà, F., Planes, J.: Detecting Disjoint Inconsistent Subformulas for Computing Lower Bounds for Max-SAT. In: Proceedings of the 21st National Conference on Artificial Intelligence (AAAI-06). vol. 1, pp. 86-91. AAAI Press (2006), event-place: Boston, Massachusetts

12. Li, C.M., Manyà, F., Planes, J.: New inference rules for max-sat. Journal of Artificial Intelligence Research 30, 321-359 (Oct 2007)

13. Martins, R., Manquinho, V., Lynce, I.: Open-wbo: A modular maxsat solver,. In: Sinz, C., Egly, U. (eds.) Theory and Applications of Satisfiability Testing - SAT 2014. pp. 438-445. Springer International Publishing, Cham (2014)

14. Narodytska, N., Bacchus, F.: Maximum satisfiability using core-guided maxsat resolution. In: Proceedings of the Twenty-Eighth Conference on Artificial Intelligence (AAAI-14). pp. 2717-2723. AAAI Press (2014)

15. P. Marques-Silva, J., A. Sakallah, K.: Grasp: A search algorithm for propositional satisfiability. Computers, IEEE Transactions on 48, 506-521 (06 1999) 